\title{
Формирование высокотемпературных сверхпроводящих слоев на границах несверхпроводящих фаз
}

\author{
() М.И. Петров ${ }^{1}$, С.И. Попков ${ }^{1,2,9}$, К.Ю. Терентьев ${ }^{1}$, А.Д. Васильев ${ }^{1,2}$ \\ ${ }^{1}$ Институт фризики им. Л.В. Киренского ФИЦ КНЦ СО РАН, Красноярск, Россия \\ ${ }^{2}$ Сибирский федеральный университет, Красноярск, Россия \\ ฯ E-mail: psi@ksc.krasn.ru
}

Поступило в Редакцию 17 марта 2020 г.

В окончательной редакции 21 мая 2020 г.

Принято к публикации 15 июля 2020 г.

\begin{abstract}
Предложен альтернативный способ синтеза образцов высокотемпературных сверхпроводников, при котором сверхпроводящие слои должны образовываться на поверхности тугоплавких гранул „зеленой фазы“ $\mathrm{Ho}_{2} \mathrm{BaCuO}_{5}$, погруженных в жидкую фазу $\mathrm{BaCuO}_{2}+\mathrm{CuO}$.
\end{abstract}

Ключевые слова: ВТСП, зеленая фаза, сверхпроводящий слой.

DOI: 10.21883/PJTF.2020.20.50148.18289

Синтез высокотемпературных сверхпроводников (ВТСП) структуры (1-2-3) обычно проводится методом стандартной керамической технологии [1-3]. В результате многократного перемалывания и отжига формируется искомая ВТСП-фаза. Было отмечено [4-6], что при синтезе ВТСП достаточно легко может образовываться побочная несверхпроводящая фаза - так называемая „зеленая фаза“ с химической формулой $R e_{2} \mathrm{BaCuO}_{5}$, где $R e-\mathrm{Y}, \mathrm{Sm}, \mathrm{Eu}, \mathrm{Gd}, \mathrm{Dy}, \mathrm{Ho}, \mathrm{Er}, \mathrm{Tm}, \mathrm{Yb}$ [5,7-9]. Cyществует метод роста из расплава (так называемый melt growth process - MG $[10,11])$, который обеспечивает лучшее спекание гранул в объемных сверхпроводниках, что приводит к увеличению плотности критического тока по сравнению с таковой для традиционно полученных ВТСП. В процессе $\mathrm{MG}[10,11]$ ВТСП нагревают до температуры, превышающей $1000^{\circ} \mathrm{C}$ (выше перитектической температуры соединения), где фаза ВТСП разлагается на твердую фазу $R_{2} \mathrm{BaCuO}_{5}$ и жидкую фазу, состоящую из $\mathrm{BaCuO}_{2}+\mathrm{CuO}$. Затем систему охлаждают, и твердая фаза $R_{2} \mathrm{BaCuO}_{5}$ подвергается перитектической реакции с жидкими фазами для повторного образования соединения ВТСП $[10,11]$.

В настоящей работе на основе метода $\mathrm{MG}[10,11]$ предложен способ получения несвязанных сверхпроводящих гранул в поликристалле, для этого к соединению $R e_{2} \mathrm{BaCuO}_{5}(R e=\mathrm{Y}$ или Но) необходимо добавить соединение $\mathrm{BaCuO}_{2}+\mathrm{CuO}$. Качественные несверхпроводящие соединения $\mathrm{Y}_{2} \mathrm{BaCuO}_{5}, \mathrm{Ho}_{2} \mathrm{BaCuO}_{5}$ и $\mathrm{BaCuO}_{2}$ получают отдельно, а затем методом твердофазного синтеза получают ВТСП-фазу. При этом синтез проводится при температуре $880^{\circ} \mathrm{C}$, что значительно ниже температур, указанных в работах $[10,11]$. При такой температуре не происходит разложения исходных составов, а ВТСП-фаза начинает образовываться на поверхности гранул $\mathrm{Y}_{2} \mathrm{BaCuO}_{5}$ или $\mathrm{Ho}_{2} \mathrm{BaCuO}_{5}$ более тугоплавкой „зеленой фазы“, помещенных в жидкую фазу $\mathrm{BaCuO}_{2}+\mathrm{CuO}$. Используя асимметричное молярное соотношение „зеленой фазы“ $\mathrm{Ho}_{2} \mathrm{BaCuO}_{5}$ и соединения $\mathrm{BaCuO}_{2}+\mathrm{CuO}$ (например, в соотношении 15:75 mol.\%), мы заведомо отклоняемся от стехиометрии ВТСП со структурой (1-2-3) и получаем в объеме поликристалла гранулы $\mathrm{Ho}_{2} \mathrm{BaCuO}_{5}$, на поверхности которых сформировался слой ВТСП $\mathrm{HoBa}_{2} \mathrm{Cu}_{3} \mathrm{O}_{7}$. Такой способ получения ВТСП-слоев в объеме поликристалла может быть применен для формирования на поверхности поликристаллического соединения $\mathrm{Ho}_{2} \mathrm{BaCuO}_{5}$ сверхпроводящих слоев различной конфигурации, что перспективно, например, для изготовления СВЧ полоснопропускающих фильтров с высокой добротностью, востребованной современной радиоэлектроникой. Детали указанного исследования будут опубликованы позже.

Однофазные несверхпроводящие соединения $\mathrm{Y}_{2} \mathrm{BaCuO}_{5}, \mathrm{Ho}_{2} \mathrm{BaCuO}_{5}$ и $\mathrm{BaCuO}_{2}$ были приготовлены методом твердофазного синтеза по стандартной керамической технологии из высокочистых оксидов $\mathrm{Ho}_{2} \mathrm{O}_{3}, \mathrm{BaCO}_{3}, \mathrm{CuO}$ на воздухе. Для $\mathrm{BaCuO}_{2}$ максимальная температура синтеза составляла $850^{\circ} \mathrm{C}$, $\mathrm{a}$ для соединений $\mathrm{Y}_{2} \mathrm{BaCuO}_{5}, \mathrm{Ho}_{2} \mathrm{BaCuO}_{5} \sim 1200^{\circ} \mathrm{C}$. Рентгеноструктурный анализ проводился на порошковом дифрактометре Bruker D8Advance (Bruker AXS). Полученные рентгенограммы соединений $\mathrm{Y}_{2} \mathrm{BaCuO}_{5}$, $\mathrm{Ho}_{2} \mathrm{BaCuO}_{5}$ и $\mathrm{BaCuO}_{2}$ совпадают с данными кристаллографической базы данных pdf@81-800, pdf@38-1402, и можно однозначно заключить, что синтезированные соединения по структуре соответствуют искомым и не содержат дополнительных фаз.

Соединения $\mathrm{Y}_{2} \mathrm{BaCuO}_{5}, \mathrm{BaCuO}_{2}$ и $\mathrm{CuO}$ были использованы при синтезе образцов с целью получения искомой ВТСП-фазы структуры $\mathrm{YBa}_{2} \mathrm{Cu}_{3} \mathrm{O}_{7}$ (1-2-3). Для этого из полученных соединений были синтезированы образцы при температуре $880^{\circ} \mathrm{C}$ с разным временем спекания: 6, 20, $200 \mathrm{~min}$. Получение ВТСП происходило по следующей схеме:

$$
\mathrm{Y}_{2} \mathrm{BaCuO}_{5}+3 \mathrm{BaCuO}_{2}+2 \mathrm{CuO} \rightarrow 2 \mathrm{YBa}_{2} \mathrm{Cu}_{3} \mathrm{O}_{7} .
$$




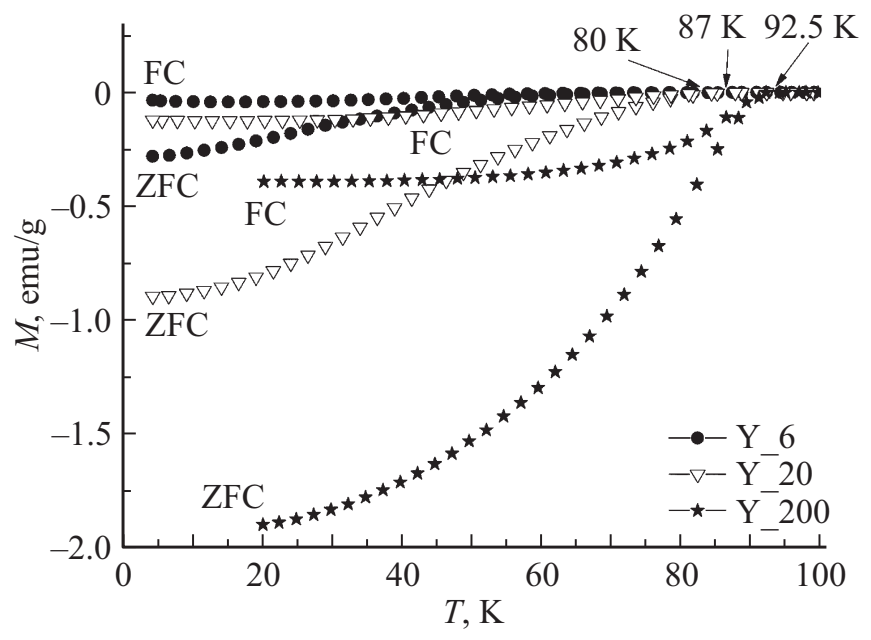

Рис. 1. Температурные зависимости намагниченности образцов Y_6, Y_20, Y_200, измеренные в режимах ZFC, FC во внешнем магнитном поле $H=50$ Ое.

По той же схеме (1) был синтезирован образец на основе ,зеленой фазы“ с Но, содержащий 15 mol.\% $\mathrm{Ho}_{2} \mathrm{BaCuO}_{5}$.

В работе приняты следующие обозначения: Y_x - образцы ожидаемого состава $\mathrm{YBa}_{2} \mathrm{Cu}_{3} \mathrm{O}_{7}$, синтезированные по схеме (1), где $x=6,20,200$ обозначает время отжига образца в минутах при температуре $880^{\circ} \mathrm{C}$; Но_0.15 образец, синтезированный по схеме (1), но содержащий только $15 \mathrm{~mol} \% \mathrm{Ho}_{2} \mathrm{BaCuO}_{5}$. Дифференциальнотермический анализ для соединения $\mathrm{BaCuO}_{2}+\mathrm{CuO}$ показал значение температуры плавления соединения $T_{m}=811.43^{\circ} \mathrm{C}$, которое значительно ниже температуры плавления соединения $\operatorname{Re}_{2} \mathrm{BaCuO}_{5}\left(\sim 1200^{\circ} \mathrm{C}\right)[6,8]$. Проведение синтеза при $T=880^{\circ} \mathrm{C}$ реализует ситуацию, при которой тугоплавкие гранулы ,зеленой фазы“ будут помещены в жидкую фазу $\mathrm{BaCuO}_{2}+\mathrm{CuO}$. При этом синтез ВТСП должен происходить на границах гранул „зеленой фазы“. Так, в работе [12] показано, что в зоне реакции на границе раздела диффузионной пары $\mathrm{BaCuO}_{2} / \mathrm{Y}_{2} \mathrm{BaCuO}_{5}$ наблюдалось формирование сверхпроводящего слоя $\mathrm{YBa}_{2} \mathrm{Cu}_{3} \mathrm{O}_{7-\delta}$.

Температурные зависимости намагниченности синтезированных образцов получены в режимах $\mathrm{ZFC}$ и $\mathrm{FC}$ в магнитном поле $H=50 \mathrm{Oe}$, зависимости $M(H)$ измерены при температуре $4.2 \mathrm{~K}$ на VSM-магнитометре установки PPMS (Quantum Design) в магнитных полях от -90 до $90 \mathrm{kOe}$.

На температурных зависимостях намагниченности образцов Y_6, Y_20, Y_200 наблюдается диамагнитный отклик как в ZFC-режиме, так и в FC-режиме. Температуры сверхпроводящих переходов соответствуют значениям $T=80,87,92.5 \mathrm{~K}$ для образцов Y_6, Y_20, Y_200 соответственно (рис. 1). На петлях гистерезиса $M(H)$ указанных образцов также наблюдается диамагнитный отклик (рис. 2). Из литературы известно, что значения диамагнитного отклика ВТСП $\mathrm{YBa}_{2} \mathrm{Cu}_{3} \mathrm{O}_{7}$ могут достигать $15-20 \mathrm{emu} / \mathrm{g}$ при $T=4.2 \mathrm{~K}$ [13]. На вставке к рис. 2 приведены для сравнения зависимости $M(H)$ образца Y_200 и объемного поликристаллического ВТСП $\mathrm{YBa}_{2} \mathrm{Cu}_{3} \mathrm{O}_{7}$ из работы [13]. Таким образом, предложенный способ получения ВТСП-слоев в объеме поликристалла позволил получить ВТСП $\mathrm{YBa}_{2} \mathrm{Cu}_{3} \mathrm{O}_{7}$, по свойствам сравнимый с лучшими поликристаллами такого состава, где ВТСП-слои начинают образовываться на границах тугоплавкого соединения $\mathrm{Y}_{2} \mathrm{BaCuO}_{5}$.

На рис. 3 представлены зависимости $M(T)$ образцов Но_0.15 (правая шкала) и Y_200 (левая шкала), на вставке к рисунку приведена зависимость $M(H)$ для образца Но_0.15. Из зависимости $M(T)$ видно, что температура сверхпроводящего перехода $\left(T_{c}\right)$ для образца Но_0.15 совпадает с $T_{c}$ образца Y_200, однако диамагнитный отклик образца Но_0.15 примерно в 10 раз ниже. Из зависимости $M(H)$ видно, что диамагнитный отклик исчезает при значении магнитного поля около $400 \mathrm{Oe}$ Значение $15 \mathrm{~mol} \%$ содержания $\mathrm{Ho}_{2} \mathrm{BaCuO}_{5}$ в объеме образца находится ниже порога протекания, и можно говорить об отдельных невзаимодействующих гранулах в поликристаллической несверхпроводящей матрице, на поверхности которых происходит синтез ВТСП-слоев. Увеличение объема сверхпроводящей фазы из несверхпроводящих компонентов наблюдалось в композитах $\mathrm{La}_{2} \mathrm{CuO}_{4}+\mathrm{La}_{1.56} \mathrm{Sr}_{0.44} \mathrm{CuO}_{4}$ [14]. Авторам работы [14] удалось оценить толщину сверхпроводящего слоя, которая составила несколько десятков нанометров.

На основе приведенных выше результатов магнитных измерений можно заключить, что в синтезированных образцах Y_6, Y_20, Y_200 значения температуры сверхпроводящего перехода $T_{c}$ увеличиваются с ростом

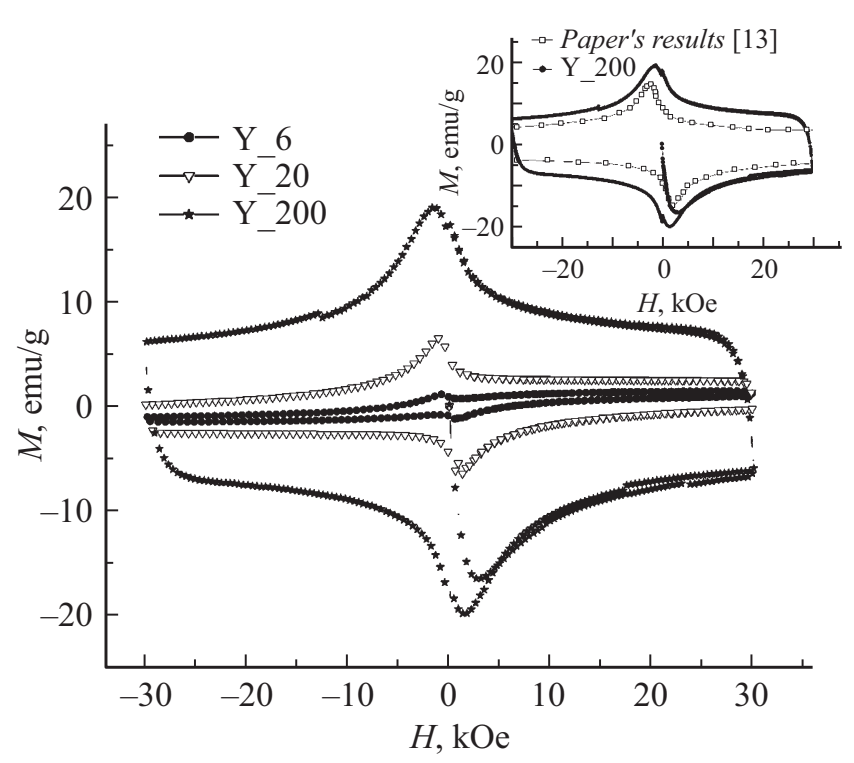

Рис. 2. Полевые зависимости намагниченности образцов Y_6, Y_20, Y_200 при температуре $T=4.2 \mathrm{~K}$. На вставке представлено сравнение зависимости $M(H)$ образца Y_200 с зависимостью $M(H)$ объемного поликристаллического иттриевого ВТСП из работы [13]. 


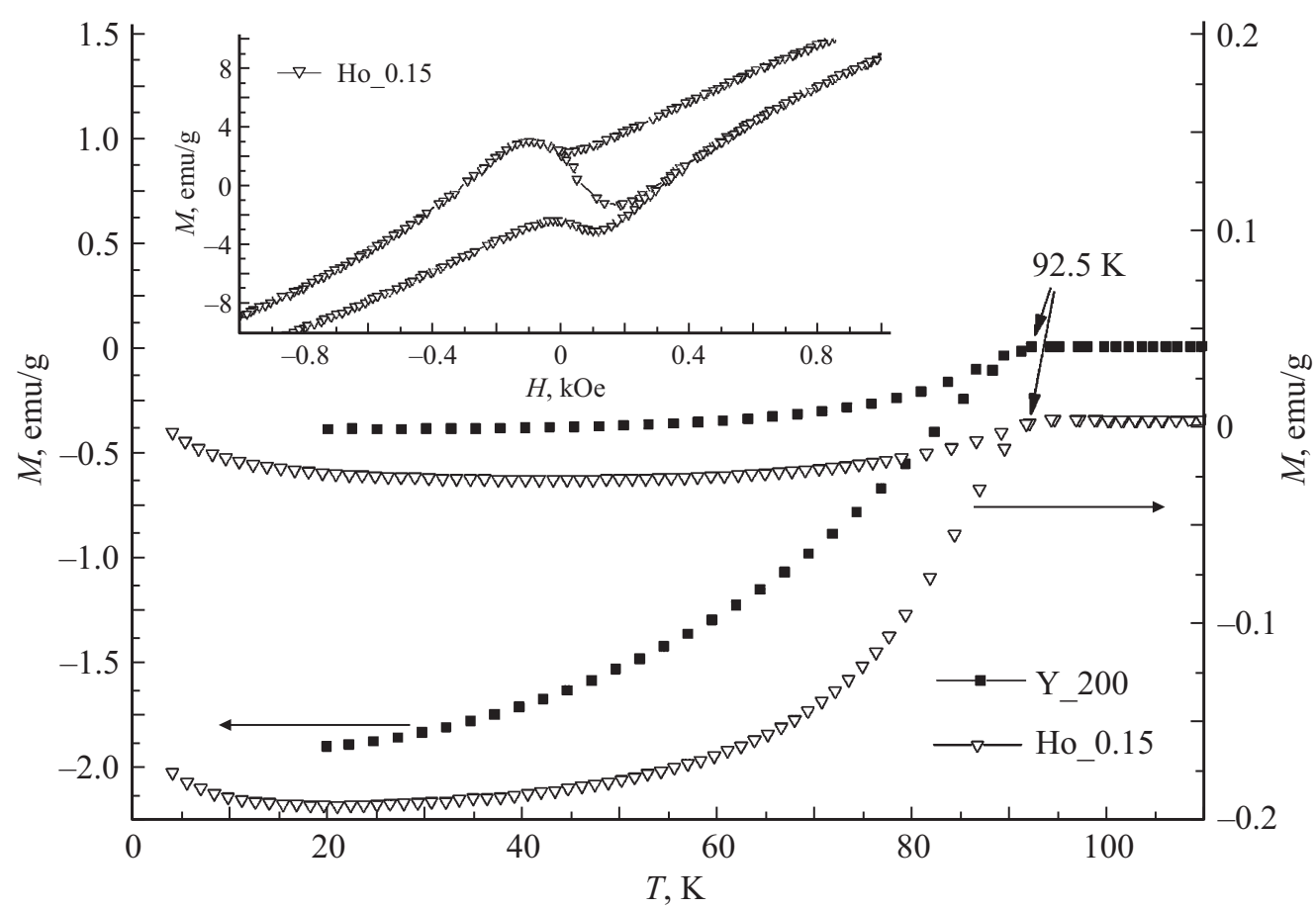

Рис. 3. Температурные зависимости намагниченности образца Но_0.15 (правая ось) и Y_200 (левая ось), измеренные в режимах $\mathrm{ZFC}, \mathrm{FC}$ во внешнем магнитном поле $H=50 \mathrm{Oe}$.

времени отжига от 80 до $87 \mathrm{~K}$ для образцов Y_6, Y_20 и до $92.5 \mathrm{~K}$ при длительности отжига $200 \mathrm{~min}$ для образца Y_200. Результаты магнитных измерений для образца Y_200 подтверждают формирование ВТСП структуры (1-2-3), диамагнитные свойства которого сравнимы с аналогичными свойствами лучших поликристаллических ВТСП, полученных по стандартной керамической технологии.

Для образца Но_0.15 значение $T_{c}$ равно $92.5 \mathrm{~K}$, что указывает на формирование ВТСП-фазы структуры (1-2-3), однако диамагнитный сигнал при гелиевых температурах в 10 раз ниже, чем для образца Y_200. Поскольку температура плавления соединений $\mathrm{Y}_{2} \mathrm{BaCuO}_{5}$ и $\mathrm{Ho}_{2} \mathrm{BaCuO}_{5}$ более $1200^{\circ} \mathrm{C}$ [6], что намного выше температуры плавления соединения $\mathrm{BaCuO}_{2}+\mathrm{CuO}$, в исследуемых образцах сверхпроводящие слои должны начинать формироваться на поверхности тугоплавких гранул $\mathrm{Ho}_{2} \mathrm{BaCuO}_{5}$ или $\mathrm{Y}_{2} \mathrm{BaCuO}_{5}$, погруженных в жидкую фазу $\mathrm{BaCuO}_{2}+\mathrm{CuO}$.

Из-за асимметричного молярного соотношения исходных ингредиентов в образце Но_0.15 ВТСП-фаза начинает формироваться в виде слоев на поверхности тугоплавких гранул фазы $\mathrm{Ho}_{2} \mathrm{BaCuO}_{5}$ и представляет собой набор невзаимодействующих ВТСП-гранул в несверхпроводящей поликристаллической матрице.

\section{Благодарности}

Результаты получены с использованием оборудования Красноярского регионального центра коллективного пользования ФИЦ КНЦ СО РАН: дифрактометра Bruker D8Advance (Bruker AXS) и вибрационного магнитометра установки PPMS (Quantum Design).

Авторы благодарят Д.М. Гохфельда за замечания при обсуждении результатов.

\section{Конфликт интересов}

Авторы заявляют, что у них нет конфликта интересов.

\section{Список литературы}

[1] Wu M.K., Ashburn J.R., Torng C.J., Hor P.H., Meng R.L., Gao L., Huang Z.J., Wang Y.Q., Chu C.W. // Phys. Rev. Lett. 1987. V. 58. P. $908-910$.

[2] Cava R.J., van Dover R.B., Batlogg B., Rietmann E.A. // Phys. Rev. Lett. 1987. V. 58. P. 408-410.

[3] Aleksandrov K.S., Vasilyev A.D., Zwegintsev S.A., Petrov M.I., Khrustalev B.P. // Physica C. 1988. V. 156. P. 249-250.

[4] Levitin R.Z., Mill B.V., Moshchalkov V.V., Samarin N.A., Snegirev V.V., Zoubkova J. // J. Magn. Magn. Mater. 1990. V. 90-91. P. 536-540.

[5] Puri M., Marrelli S., Bear J., Kevan L. // Appl. Magn. Res. 1990. V. 1. P. 509-524.

[6] Baranchikov A.E., Grigorashev D.I., Sokolov S.V., Olenikov N.N. // Inorgan. Mater. 1997. V. 33. P. 946-950.

[7] Whatkins S.F., Fronczek F.R., Wheelock K.S., Goodrich R.G., Hamilton W.D., Johnson W.W. // Acta Cryst. C. 1988. V. 44. P. 3-6.

[8] Ovsyanikov A.K., Golosovsky I.V., Zobkalo I.A., Mirebeau I. // J. Magn. Magn. Mater. 2014. V. 353. P. 71-75. 
[9] Zhang Y., Li H., Wang J., Li X., Ren Z., Wilde G. // Ceram. Int. 2018. V. 44. P. 1991-1994.

[10] Durrell J.H., Dennis A.R., Jaroszyski J., Ainslie M.D., Palmer K.G.B., Shi Y.H., Campbell A.M., Hull J., Strasik M., Hellstrom E.E., Cardwell D.A. // Supercond. Sci. Technol. 2014. V. 27. P. 082001.

[11] Superconductivity. Application today and tomorrow / Ed. M. Miryala. N.Y.: Nova Science Publ. Inc., 2016. P. 1-37.

[12] Kalanda N.A., Trukhan V.M., Marenkin S.F. // Inorgan. Mater. 2002. V. 38. P. 723-729.

[13] Semenov S.V., Balaev D.A. // J. Supercond. Novel Mag. 2019. V. 32. P. 2409-2419.

[14] Bykov A.A., Terent'ev K.Yu., Gokhfeld D.M., Savitskaya N.E., Popkov S.I., Petrov M.I. // J. Supercond. Novel Mag. 2019. V. 32. P. $3797-3802$. 